\title{
Five ways to model active processes in elastic solids: active forces, active stresses, active strains, active fibers, and active metrics.
}

\author{
A. Goriely
}

Mathematical Institute, University of Oxford, Oxford, UK

\begin{abstract}
"A particular point to be emphasized is that while continuum mechanics was for a long time reserved to the study of inert matter, this new mechanics of materials now dares to attack the landscape of living matter in the framework of biomechanics and mechano-biology for the study of growth, resorption, aging, remodeling, and morphogenesis... Non-linear elasticity was in some sense saved from oblivion by its useful applications in biomechanics."
\end{abstract}

Gérard A. Maugin [1]

\section{Introduction}

An important contribution of Gérard Maugin was his study of anelasticity $[2,3]$. It is well appreciated that in biological systems, anelasticity manifests itself through growth and remodeling [4] and Maugin and his collaborators have helped build a general theory suitable to model such fundamental processes [5, 6]. Yet another important manifestation of anelasticity is the active processes found in all living systems. These include muscular fiber contraction [7] or the traction exerted by neuronal growth cones [8]. They are essential for all aspects of life [9] from motility [10] and cell division [11] at the cellular scale to muscular motion at the organ level [12]. We distinguish active processes from growth and remodeling as those anelastic processes that only affect temporarily the reference configuration. Growth is associated with a change of total mass and remodeling is associated with

Email address: goriely@maths .ox.ac.uk (A. Goriely) a change in the material properties. In contrast, active processes take place on shorter time scales and are usually associated with motility and muscular motion. Note, however, that this distinction is a practical one and not a formal one. Indeed, motion in plants is accomplished by lengthening and changes in material properties rather than fast muscular contraction.

In order to exist, these processes must be developed actively within the material by spending energy. At the cellular level, the term active material mostly refers to filament networks containing molecular motors such as myosin [13]. These force-generating units are attached to filaments and consume adenosine triphosphate (ATP) to change their molecular configurations which result in the relative sliding and tensioning of these filaments. In an active fluid system, such as bacterial suspension responding to chemotactic stimuli, these forces create a bath of interacting self-propelled microorganisms which are known to undergo complex dynamics and pattern formation as a result of the interplay between active force generation and hydrodynamic interactions [14].

Mechanically, these forces are not due to the action of external loads or external body forces and are not present in a typical engineered material. From a modeling perspective, they can be introduced in the governing equations at different levels. Different forms have been presented in the literature. Here, we present a mini-review of the different ways to model active processes in an elastic solid. Assuming that active forces do not generate torques, their presence does not alter the basic governing equations expressing the balance of linear and angular momenta, that can be expressed in the current configura- 
tion as

$$
\operatorname{div} \mathbf{T}+\rho \mathbf{b}=\rho \dot{\mathbf{v}}, \quad \mathbf{T}^{\top}=\mathbf{T},
$$

where, as usual, $\rho$ is the density, $\mathbf{v}$ the velocity of a material point, $\mathbf{T}$ is the Cauchy stress tensor and $\mathbf{b}$ a body-force density.

\section{Active forces}

The first obvious way to include active forces in a material is to use body forces and decompose them as

$$
\mathbf{b}=\mathbf{b}^{\mathrm{e}}+\mathbf{b}^{\mathrm{a}}
$$

where $\mathbf{b}^{\mathrm{e}}$ represents the external body forces (such as gravity) and $\mathbf{b}^{\mathrm{a}}$ is an active body force term. The general form of this term is not quite obvious. What is the force actively generated by filament sliding or point forces? At the microscale, the forces developed by a cell due to division, apoptosis, or motion act locally at the points of contact with the elastic medium [15]. Assuming that there are $N$ such discrete points in the medium, these forces can be represented as local force dipoles so that

$$
\mathbf{b}^{\mathrm{a}}=\operatorname{div} \mathbf{Q} \quad \text { where } \quad \mathbf{Q}=\sum_{i=1}^{N} \mathbf{D}^{(i)} \delta\left(\mathbf{x}-\mathbf{x}^{(i)}\right),
$$

represents the body force stress due to the force dipoles. Here, $\mathbf{D}^{(i)}$ is a symmetric tensor and $\delta\left(\mathbf{x}-\mathbf{x}^{(i)}\right)$ is Dirac's distribution centered at the point of contact $\mathbf{x}^{(i)}$. By analogy, the effects of these force dipoles can be treated in the same way as electric charges in a polarizable medium, defects in solids, or interacting active molecules in nematic liquid crystals [16].

In linear elasticity, a suitable Green's function can be introduced to represent the effects of highly localized forcing terms for the Cauchy equation (1). However, in nonlinear elasticity, the presence of distributions as forcing terms cannot be easily handled unless a weak form of the equations is used within a computational framework [17]. From a mathematical perspective, it may therefore be more suitable to think of forces as acting over a finite volume and directly work with an active stress tensor. We remark that the form (3) implies that $\mathbf{Q}$ is a particular form of an active stress tensor as we explains next.

\section{Active stresses}

Rather than postulating the existence of active forces, the presence of active mechanisms within a continuum tissue can be modeled by an active stress. In this approach, the Cauchy stress tensor $\mathbf{T}$ is split into two components:

$$
\mathbf{T}=\mathbf{T}^{\mathrm{e}}+\mathbf{T}^{\mathrm{a}}
$$

where $\mathbf{T}^{\mathrm{e}}$ represents the stress produced by the elastic response of the material in the absence of active mechanisms. The choice of an additive decomposition between active and elastic components is made out of convenience. For a hyperelastic material with a strain-energy density $W=W(\mathbf{F})$, the elastic stress is given constitutively by

$$
\mathbf{T}^{\mathrm{e}}=J^{-1} \mathbf{F} \frac{\partial W}{\partial \mathbf{F}}-p \mathbf{1}
$$

where $J=\operatorname{det}(\mathbf{F})$ equals one for an incompressible material and $p=0$ for compressible material. The active stress $\mathbf{T}^{\mathrm{a}}$ is a symmetric tensor postulated phenomenologically to represent the effect of local contractions. Since contraction is typically localized and directed it will be, in general, both inhomogeneous and anisotropic. In the simplest case, the tensor $\mathbf{T}^{\mathrm{a}}$ can be taken as a multiple of the identity, corresponding to a localized change in pressure due to contractile forces [18]. The stress generated may depend on the deformation itself, therefore, we expect the active stress to be a function of the deformation tensor $\mathbf{T}^{\mathrm{a}}=\mathbf{T}^{\mathrm{a}}(\mathbf{F})$. Along these lines, different models have been proposed in connection with heart muscle contraction [19, 20, 21]. It follows immediately from this description and (2) that this active stress tensor defines an active body force through the relationship

$$
\rho \mathbf{b}^{\mathrm{a}}=\operatorname{div} \mathbf{T}^{\mathrm{a}}
$$

However, it should be noted that not all body forces can be written as stress tensors. Stresses act in a material at short-ranges through tractions and are not suitable to describe the effect of long-range forces, hence the decomposition (2) between external (long-range) and internal (active) body forces.

The active stress approach has been used extensively in soft-tissue biomechanics and in particular in the modeling of heart contractions [22]. It is usually assumed that the active stress is in the direction of the muscle fibers and a 
function of both the stretch (due to the presence of stretchactivated channels in cardiomyocytes [23]) and calcium concentration controlling the dynamics of the muscular activity $[24,25,26]$.

\section{Active strains}

Muscular contraction or relative sliding of actin filaments can be interpreted as a temporary internal remodeling of the continuum. This remodeling can be modeled by the methods used in the theory of growth [27, 28] as a change in the reference configuration. In this approach $[29,30]$, the deformation gradient is decomposed multiplicatively as is done in morphoelasticity

$$
\mathbf{F}=\mathbf{F}^{\mathrm{e}} \mathbf{F}^{\mathrm{a}}
$$

where $\mathbf{F}^{\mathrm{e}}$ and $\mathbf{F}^{\mathrm{a}}$ are the elastic and active deformation tensors, respectively. Since, typically we do not expect a change in volume during an active response, we restrict the choice of active strains so that $\operatorname{det} \mathbf{F}^{\mathrm{a}}=1$. The advantage of this representation is that it can be directly linked to the relative displacement of materials taking place during contraction. Rather than imposing an internal force, it imposes an internal displacement.

From a theoretical point of view, this decomposition is exactly the one used in morphoelasticity and in the theory of anelasticity developed by Maugin and collaborators [3]. Therefore, the entire machinery developed to understand anelastic problems can be used directly to understand the active stresses generated by active strains with the appropriate change of terminology. It has also been argued that it presents several advantages from a theoretical and computational point of view [31, 32].

\section{Active metrics}

Another possible method to quantify active reorganization is by changing the underlying metric for the reference manifold. These ideas borrowed from differential geometry are popular in the physics community [33, 34] and have also been used successfully in nonlinear anelasticity $[35,3,36]$. The basic idea is that during an active contraction process, the material distance between point is shortened. Hence, the reference configuration changes and, as a consequence, internal stresses build up. Therefore, active processes can be modeled by postulating an active metric $\mathbf{G}^{\mathrm{a}}$. This metric can then be used in a general theory of elastic material with arbitrary metrics [37, 38] to compute the stresses generated by the active process.

There is a well-know connection between anelastic strains and anelastic metrics that identify the new metric with the right Cauchy-Green tensor associated with the anelastic part of the deformation tensor. In the case of active process, the active metric is related to the active strains by

$$
\mathbf{G}^{\mathrm{a}}=\left(\mathbf{F}^{\mathrm{a}}\right)^{\top} \mathbf{F}^{\mathrm{a}}
$$

Note that the specification of an active metric $\mathbf{G}^{\mathrm{a}}$ does not imply a unique choice of an active strain since there is a well-known arbitrary rotational freedom (i.e. both $\mathbf{F}^{\mathrm{a}}$ and $\mathbf{R F}^{\mathrm{a}}$ lead to the same metric for all tensors $\left.\mathbf{R} \in S O(3)\right)$.

\section{Active fibers}

Another way to model active growth is at the level of the strain-energy density, either by using pseudo-elasticity [39] or by assuming that the zero-strain energy along contracting fibers differs from the zero-strain state for the rest of the tissue [40, 41]. As an example, following the theory of invariants for anisotropic materials [42], we assume that the strain-energy density only depends on the invariants $I_{1}, I_{2}$ for the isotropic part and $I_{4}$ for the anisotropic part, so that

$$
W=W\left(I_{1}-3, I_{2}-3, I_{4}-v\right),
$$

where $I_{1}, I_{2}$ are the usual invariants of incompressible isotropic elasticity and $I_{4}=\mathbf{M} \cdot\left(\mathbf{F}^{\mathrm{T}} \mathbf{F} \mathbf{M}\right)$ represents the stretch in a fiber originally in a direction $\mathbf{M}$ by the deformation gradient $\mathbf{F}$. When $v=1$, the strain-energy has a global minimum in the absence of deformation given by $I_{1}=I_{2}=3$ and $I_{4}=1$. However, the elastic energy increases when $v \neq 1$ describing the rest state of the fibre (with $0<v<1$ describing active fiber contraction).

\section{An elementary example}

To understand how these notions can be implemented effectively, we consider a toy model: the contraction of an incompressible hyperelastic cylinder with one family 
of fibers oriented in the hoop direction. We further assume that the active process is a contraction (or relaxation) taking place in the hoop direction to mimic the effect of muscle contraction. The general case of one or two families of helical fibers as been studied by the method of active fibers in [42].

A tube of initial inner radius $A=1$ and outer radius $B>A$, and height $H$ is deformed into a tube with radii $a$ and $b$ and the same height $H$. We consider only deformation in which the cylinder is allowed to inflate while remaining cylindrical. We assume that the elastic material is incompressible and that no growth process is taking place. In cylindrical coordinates, the deformation is then $r=\sqrt{a^{2}+R^{2-} A^{2}}, \theta=\Theta, z=Z$, with position vectors $\mathbf{X}=R \mathbf{e}_{r}+Z \mathbf{e}_{z}, \mathbf{x}=\lambda R \mathbf{e}_{r}+Z \mathbf{e}_{z}$. The deformation gradient (in cylindrical coordinates) is thus $\mathbf{F}=\operatorname{Grad} \mathbf{x}=$ $\operatorname{diag}(1 / \lambda, \lambda, 1)$, with $\lambda=r / R$. Therefore, a single parameter, $a$, fully describes the radial profile of the deformation and it follows that $b(a)=B \sqrt{1+\left(\lambda_{a}^{2}-1\right) A^{2} / B^{2}}$.

Since we allow inelastic strains, we define the elastic and active strains as

$$
\mathbf{F}^{\mathrm{e}}=\operatorname{diag}\left(\alpha_{r}, \alpha_{\theta}, 1\right), \quad \mathbf{F}^{\mathrm{a}}=\operatorname{diag}(1 / \gamma, \gamma, 1),
$$

with $\alpha_{r}=1 / \alpha_{\theta}$. For illustration purpose, we will use the standard fiber-reinforcing model [43]

$$
\begin{aligned}
W & =\frac{\mu_{1}}{2}\left(I_{1}-3\right)+\frac{\mu_{4}}{4}\left(I_{4}-v\right)^{2} \\
& =\frac{1}{2} \mu_{1}\left(\alpha_{\theta}^{2}+\alpha_{r}^{2}-2\right)+\frac{1}{4} \mu_{4}\left(\alpha_{\theta}^{2}-v\right)^{2},
\end{aligned}
$$

and, allowing for (constant) active stresses in the hoop direction, the stresses are related to the strains by

$$
T_{r r}=\alpha_{r} \frac{\partial W}{\partial \alpha_{r}}-p, \quad T_{\theta \theta}=\alpha_{\theta} \frac{\partial W}{\partial \alpha_{\theta}}-p+T_{\theta \theta}^{\mathrm{a}} .
$$

For this simple problem, we note that the Cauchy equation $\operatorname{div} \mathbf{T}=0$ leads to a single equation

$$
\frac{\mathrm{d} T_{r r}}{\mathrm{~d} r}+\frac{1}{r}\left(T_{r r}-T_{\theta \theta}\right)+\rho b_{r r}^{\mathrm{a}}=0 .
$$

This equation can be integrated once over $r$, and, in the absence of external load, the equation for the remaining unknown $a$ is

$$
0=\int_{a}^{b(a)} \frac{T_{\theta \theta}-T_{r r}}{r}+\rho b_{r r}^{\mathrm{a}} \mathrm{d} r .
$$
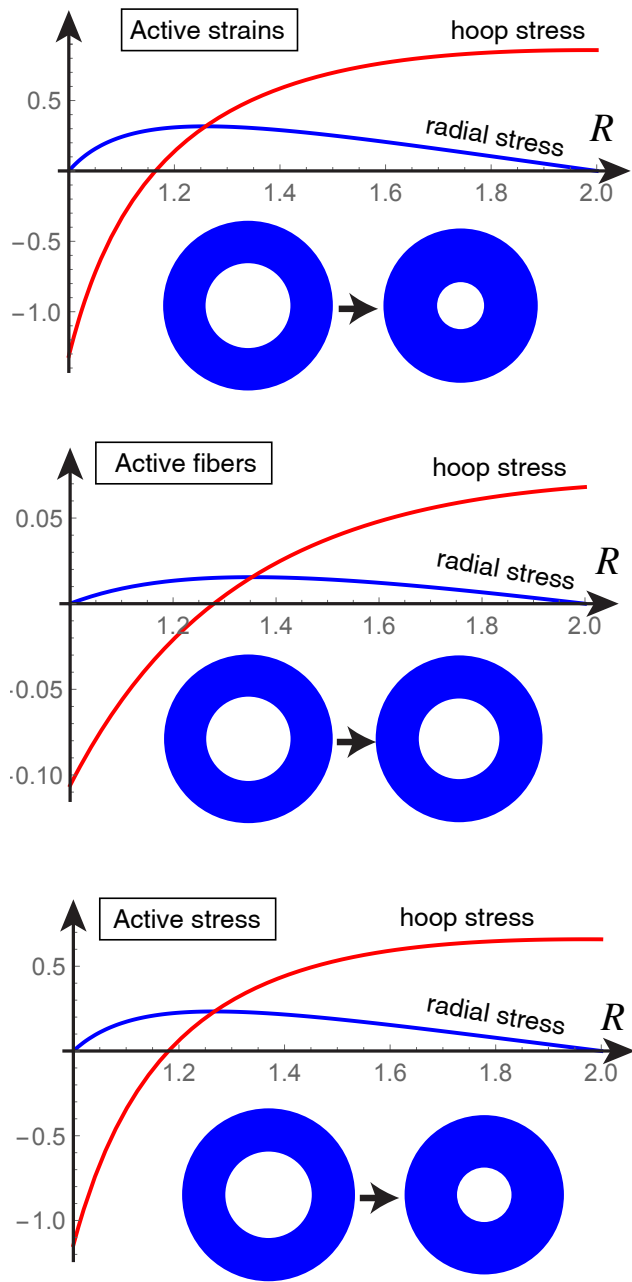

Figure 1: Comparison of three different models to include hoop active contraction in the section of a cylinder. Here $A=1, B=2, \mu_{1}=1, \mu_{4}=$ $1 / 2$ and from left to right $\gamma_{\theta}=3 / 4, v=3 / 4, T_{\theta \theta}^{\mathrm{a}}=1$ (respectively). In each case, all other active processes are removed (e.g. for active strains, we take $v=1$ and $T_{\theta \theta}^{\mathrm{a}}=0$ ). Note the difference in $y$-scale for the case of active fiber (by a factor of about 10).

Once $a$ is known, the stretches $\alpha_{\theta}$ and $\alpha_{r}$ are fully determined and so are the stresses. In Fig. 1, we compare the different models of active contractions. We note that they all lead to similar stress profiles. However, active fiber contraction does not lead to large contraction (even when taking $v=0$ ). This is due to the fact that the energy con- 
tained in the fibers may be small compared to the elastic matrix and a large extension of the fibers may be preferable to minimize the overall elastic energy. In contrast, the active strains force the contraction to happen and the material has to accommodate such large internal displacements. Active stresses can be tuned to model the effects of active strains. However, formally, the two approaches are different and there is no one-to-one correspondence.

In many physiological systems, it is often assumed that fibers cannot support compression as free fibers in a matrix would buckle [44]. An easy way to take this effect into account is to exclude from the strain-energy density, fibers that are not in tension. In that case, one replaces (11) by

$$
W=\frac{\mu_{1}}{2}\left(I_{1}-3\right)+\frac{\mu_{4}}{4}\left[I_{4}-v\right]_{+}^{2},
$$

where $[x]_{+}=x$ if $x \geq 0$ and 0 otherwise. For active radial stress or active radial strains, as given in the previous section, the fibers are always in compression. Hence the anisotropic term does not play a role and the stress profiles are independent of $\mu_{4}$. However, in the case of active fibers, the shift of ground state from 1 to $v$, implies that the fibers are active. Therefore, for large enough values of $\mu_{4}$, one recovers the same stress profile as in the case of active strains and active stress as shown in Figure 2

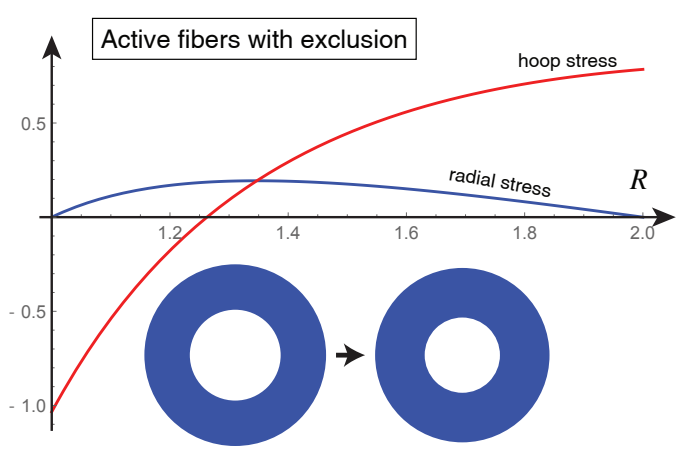

Figure 2: The case of fiber exclusion. Here $A=1, B=2, \mu_{1}=1, \mu_{4}=$ $6, v=3 / 4$.

The active stresses approach can be used to compute equivalent active forces. Taking the divergence of the active stresses lead to the active body forces: $\rho \mathbf{b}^{a}=$ $-\mathbf{e}_{r} T_{\theta \theta}^{\mathrm{a}} / r$. The active forces is in the radial direction and a function of the position $r$. Similarly, the active strains can be used to compute an active metric simply given, in the usual cylindrical coordinates by $\mathbf{G}^{\mathrm{a}}=\operatorname{diag}\left(1 / \gamma^{2}, \gamma^{2}, 1\right)$.

\section{Discussion}

The five different approaches to model active processes here are all suitable on a kinematic basis. The difficulty comes when relating the new active field to other physical and kinematic quantities. Depending on the particular application, a phenomenological law for active process is required. The formulation of this law may be easier by choosing a particular model of active process. For instance, in a tissue, with embedded muscular fibers, the contraction of these fibers may be easily represented by active fibers. If the active processes is known to impose a local force, then the active stress approach may be more suitable. If the relative displacement of neighboring elements is actively controlled, the active strain approach will be better suited. The choice between active strains or active stress is then similar to the classic choice of boundary conditions (traction versus displacement) in a regular problem of elasticity. However, the coupling with other fields may greatly change the stresses generated by the active processes and great care must be exercised when using one approach or another.

\section{Note}

The present opinion piece is an expanded and illustrated version of Challenge \#9 presented in the conclusions of [4] as one of the important open challenges in the theory of biological growth.

\section{References}

[1] Gérard A Maugin. Continuum mechanics through the twentieth century. Springer, 2013.

[2] R. Kienzler and G. A. Maugin. Configurational Mechanics of Materials. Springer, 2001.

[3] M. Epstein and G. A. Maugin. On the geometrical material structure of anelasticity. Acta Mech., 115(1):119-131, 1996.

[4] A. Goriely. The Mathematics and Mechanics of Biological Growth. Springer Verlag, New York, 2017. 
[5] S. Imatani and G. A. Maugin. A constitutive model for material growth and its application to threedimensional finite element analysis. Mech. Res. Comm., 29:477-483, 2002.

[6] M. Epstein and G. A. Maugin. Thermomechanics of volumetric growth in uniform bodies. Int. J. Plasticity, 16(7-8):951-978, 2000.

[7] D. E. Rassier, B. R. MacIntosh, and W. Herzog. Length dependence of active force production in skeletal muscle. J. Appl. Physiol., 86(5):1445-1457, 1999.

[8] Pierre Recho, Antoine Jerusalem, and Alain Goriely. Growth, collapse, and stalling in a mechanical model for neurite motility. Phys. Rev. E, 93(3):032410, 2016.

[9] K. H. Iwasa and R. S. Chadwick. Elasticity and active force generation of cochlear outer hair cells. $J$. Acoust. Soc. Am., 92(6):3169-3173, 1992.

[10] M. E. Gracheva and H. G. Othmer. A continuum model of motility in ameboid cells. Bull. Math. Biol., 66(1):167-193, 2004.

[11] S. W. Grill, J. Howard, E. Schäffer, E. H. K. Stelzer, and A. A. Hyman. The distribution of active force generators controls mitotic spindle position. Science, 301(5632):518-521, 2003.

[12] D. A. Fletcher and P. L. Geissler. Active biological materials. Annu. Rev. Phys. Chem., 60:469-486, 2009.

[13] M. J. Shelley. The dynamics of microtubule/motorprotein assemblies in biology and physics. Аnпи. Rev. Fluid Mech., 48:487-506, 2016.

[14] D. Saintillan and M. J. Shelley. Active suspensions and their nonlinear models. Abbreviation Title C.R. Phys., 14(6):497-517, 2013.

[15] J. Ranft, M. Basan, J. Elgeti, J. F. Joanny, J. Prost, and F. Jülicher. Fluidization of tissues by cell division and apoptosis. Proc. Natl. Acad. Sci. USA, 107(49):20863-20868, 2010.
[16] M. C. Marchetti, J. F. Joanny, S. Ramaswamy, T. B. Liverpool, J. Prost, M. Rao, and R. A. Simha. Hydrodynamics of soft active matter. Rev. Mod. Phys, 85(3):1143, 2013.

[17] Z. Wang, S. Rudraraju, and K. Garikipati. A threedimensional field formulation, and isogeometric solutions to point and line defects using Toupin's theory of gradient elasticity at finite strains. J. Mech. Phys. Solids, 94:336-361, 2016.

[18] K Dasbiswas, E Alster, and SA Safran. Mechanobiological induction of long-range contractility by diffusing biomolecules and size scaling in cell assemblies. Scientific reports, 6:27692, 2016.

[19] M. Böl, O. J. Abilez, A. N. Assar, C. K. Zarins, and E. Kuhl. In vitro/in silico characterization of active and passive stresses in cardiac muscle. Int. J. Multiscale Comp. Eng., 10:171-188, 2012.

[20] A. V. Panfilov, R. H. Keldermann, and M. P. Nash. Self-organized pacemakers in a coupled reaction-diffusion-mechanics system. Phys. Rev. Lett., 95(25):258104, 2005.

[21] N. P. Smith, D. P. Nickerson, E. J. Crampin, and P. J. Hunter. Multiscale computational modelling of the heart. Acta Numerica, 13:371-431, 2004.

[22] JM Guccione and AD McCulloch. Mechanics of active contraction in cardiac muscle: part iconstitutive relations for fiber stress that describe deactivation. Journal of biomechanical engineering, 115(1):72-81, 1993.

[23] Peter Kohl, Peter Hunter, and Denis Noble. Stretchinduced changes in heart rate and rhythm: clinical observations, experiments and mathematical models. Progress in biophysics and molecular biology, 71(1):91-138, 1999.

[24] Markus Böl, Stefanie Reese, Kevin Kit Parker, and Ellen Kuhl. Computational modeling of muscular thin films for cardiac repair. Computational Mechanics, 43(4):535-544, 2009.

[25] Serdar Göktepe and Ellen Kuhl. Electromechanics of cardiac tissue: A unified approach to the fully 
coupled excitation-contraction problem. PAMM, 9(1):159-160, 2009.

[26] MK Rausch, A Dam, S Göktepe, OJ Abilez, and E Kuhl. Computational modeling of growth: systemic and pulmonary hypertension in the heart. Biomech. Model. Mechanobiol., 10(6):799-811, 2011.

[27] E. K. Rodriguez, A. Hoger, and A. McCulloch. Stress-dependent finite growth in soft elastic tissue. J. Biomech., 27:455-467, 1994.

[28] M. Ben Amar and A. Goriely. Growth and instability in elastic tissues. J. Mech. Phys. Solids, 53:22842319, 2005.

[29] C. Cherubini, S. Filippi, P. Nardinocchi, and L. Teresi. An electromechanical model of cardiac tissue: Constitutive issues and electrophysiological effects. Prog. Biophys. Mol. Biol., 97(2):562-573, 2008.

[30] P. Nardinocchi and L. Teresi. On the active response of soft living tissues. Journal of Elasticity, 88(1):2739, 2007.

[31] D. Ambrosi and S. Pezzuto. Active stress vs. active strain in mechanobiology: constitutive issues. J. Elasticity, 107(2):199-212, 2012.

[32] S. Pezzuto and D. Ambrosi. Active contraction of the cardiac ventricle and distortion of the microstructural architecture. International journal for numerical methods in biomedical engineering, 30(12):1578-1596, 2014.

[33] E. Sharon, M. Marder, and H. L. Swinney. Leaves, flowers and garbage bags: Making waves. Am. Sci., 92(3):254-261, 2004.

[34] E. Efrati, Y. Klein, H. Aharoni, and E. Sharon. Spontaneous buckling of elastic sheets with a prescribed non-Euclidean metric. Physica D, 235(1-2):29-32, 2007.

[35] M. Epstein and G. A. Maugin. Geometric material structure of finite-strain elasticity and anelasticity. ZAMM, 76:125-128, 1996.
[36] A. Yavari. A geometric theory of growth mechanics. J. Nonlinear Sci., 20:781-830, 2010.

[37] S. Sadik, A. Angoshtari, A. Goriely, and A. Yavari. A geometric theory of nonlinear morphoelastic shells. J. Nonlinear Sci., pages 1-50, 2016.

[38] A. Yavari and A. Goriely. The geometry of discombinations and its applications to semi-inverse problems in anelasticity. Proc. Roy. Soc. Lond. A, 470(2169):20140403, 2014.

[39] C. Paetsch, B. A. Trimmer, and L. A. Dorfmann. A constitutive model for active-passive transition of muscle fibers. Int. J. Non-Linear Mech., 47(2):377387, 2012.

[40] A. Goriely and M. Tabor. Spontaneous rotational inversion in Phycomyces. Phys. Rev. Lett., 106(13):138103, 2011.

[41] D. E. Moulton, T. Lessinnes, S. O'Keeffe, L. A. Dorfmann, and A. Goriely. The elastic secrets of the chameleon tongue. Proc. Roy. Soc. Lond. A, 472(2188):20160030, 2016.

[42] A. Goriely and M. Tabor. Rotation, inversion and perversion in anisotropic elastic cylindrical tubes and membranes. Proc. Roy. Soc. Lond. A, 469(2153), 2013.

[43] N. Triantafyllidis and R. Abeyaratne. Instabilities of a finitely deformed fiber-reinforced elastic material. J. Appl. Mech., 50:149-156, 1983.

[44] G. A. Holzapfel and R. W. Ogden. Constitutive modelling of arteries. Proc. Roy. Soc. Lond. A, 466(2118):1551-1597, 2010. 